\title{
New detections of Mn, Ti and Mg in damped Ly $\alpha$ systems: Toward reconciling the dust/nucleosynthesis degeneracy ${ }^{\star}$
}

\author{
M. Dessauges-Zavadsky ${ }^{1,2}$, J. X. Prochaska ${ }^{3,4}$, and S. D'Odorico ${ }^{1}$ \\ ${ }^{1}$ European Southern Observatory, Karl-Schwarzschildstr. 2, 85748 Garching bei München, Germany \\ e-mail: mdessaug@eso.org; sdodoric@eso.org \\ 2 Observatoire de Genève, 1290 Sauverny, Switzerland \\ 3 The Observatories of the Carnegie Institute of Washington, 813 Santa Barbara Street, Pasadena, CA 91101, USA \\ e-mail: xavier@ociw.edu \\ ${ }^{4}$ Hubble Fellow
}

Received 12 February 2002 / Accepted 4 June 2002

\begin{abstract}
We have combined new high resolution UVES-VLT observations of a sample of four damped Ly $\alpha$ systems (DLAs) at redshifts between $z_{\mathrm{abs}}=1.7-2.5$ with the existing HIRES-Keck spectra to undertake a comprehensive study of their physical conditions and their abundances of up to 15 elements. In this paper, we present abundance measurements for $\mathrm{Mn}, \mathrm{Ti}$ and $\mathrm{Mg}$ which are among the first presented in the literature at these redshifts. We confirm the underabundance of Mn with respect to $\mathrm{Fe}$ as observed in lower redshift DLAs and a trend with decreasing metallicity very similar to the one observed in Galactic metal-poor stars. This agreement between the Mn/Fe ratios in DLAs and in Galactic stars suggests that the DLAs and the Milky Way share some similarities in their star formation histories. However, these similarities must be cautiously interpreted and investigated in light of all of the elements observed in the DLAs. We have obtained a first measurement and a significant upper limit of the Ti abundance at $z_{\text {abs }} \sim 2$ from the Ti II lines at $\lambda_{\text {rest }}>3000 \AA$, and we discuss how the relative abundance of this highly depleted element can be used to prove unambiguously any enhancement of the abundance of the $\alpha$-elements relative to Fe-peak elements. We present the abundances of Mg for two DLAs in addition to the single $\mathrm{Mg}$ measurement existing in the literature. Contrary to the trend expected from differential depletion, the $[\mathrm{Mg} / \mathrm{Si}, \mathrm{S}]$ ratios tend to be over-solar. The effect is at the level of the measurement errors, but worth investigating in a larger sample because it could be suggestive of a peculiar nucleosynthesis effect.
\end{abstract}

Key words. cosmology: observations - galaxies: abundances - galaxies: evolution - quasars: absorption lines

\section{Introduction}

A measure of chemical enrichment in high redshift galaxies can be obtained through the study of absorption line systems in quasars, specifically via damped Ly $\alpha$ systems (DLAs). These systems with $N(\mathrm{H} \mathrm{I})>2 \times 10^{20} \mathrm{~cm}^{-2}$ dominate the neutral hydrogen content of the Universe and are likely the protogalactic gas reservoirs for the formation of the majority of stars today (e.g. Wolfe et al. 1995). They provide the best opportunity to measure accurately the chemical abundances of many elements for a variety of galactic systems spanning a wide redshift interval. By comparing these abundance measurements with the chemical evolution models and abundance patterns of our Galaxy and nearby galaxies, one can infer information on the star formation history and galaxy evolution of these distant objects.

Send offprint requests to: M. Dessauges-Zavadsky,

e-mail: mdessaug@eso.org

* Based on the UVES observations collected during the ESO prog. ID No. 67.A-0022 at the VLT/Kueyen telescope, Paranal, Chile.
In particular, abundances of the $\alpha$-elements (e.g. Si, O, S, $\mathrm{Ar}$ ) relative to the $\mathrm{Fe}$-peak elements (e.g. $\mathrm{Fe}, \mathrm{Cr}, \mathrm{Ni}$ ) are of great importance for defining the chemical evolution history. Being produced mainly by type II and type Ia supernovae ( $\mathrm{SNe}$ ) with relatively different timescales $-<2 \times 10^{7}$ ( $\alpha$-elements) and $10^{8}-10^{9}$ yrs (Fe-peak elements) - respectively, the $\alpha / \mathrm{Fe}$ ratios are strongly dependent on the lifetimes of the element progenitor, whereas $[\mathrm{Fe} / \mathrm{H}]^{1}$ depends on the star formation rate (Tinsley 1979; Matteucci 2001). Therefore, the $[\alpha / \mathrm{Fe}]$ versus $[\mathrm{Fe} / \mathrm{H}]$ relation is a strong function of the star formation history.

Unfortunately this scheme to discriminate between different star formation histories has led to contradictory conclusions and interpretations of the observed relative abundances of the damped Ly $\alpha$ systems (e.g. Lu et al. 1996; Centurión et al. 2000). The principle difficulty is to disentangle nucleosynthetic contributions from dust depletion effects. Because we are studying gas-phase abundances in DLAs, the measured abundances may not represent the intrinsic composition of the system if part of the elements is removed from the gas to

\footnotetext{
${ }^{1}[\mathrm{X} / \mathrm{H}] \equiv \log [N(\mathrm{X}) / N(\mathrm{H})]_{\text {DLA }}-\log [N(\mathrm{X}) / N(\mathrm{H})]_{\odot}$.
} 
Table 1. Metal abundances.

\begin{tabular}{|c|c|c|c|c|c|c|c|}
\hline Quasar & $z_{\text {abs }}$ & {$[\mathrm{Fe} / \mathrm{H}]$} & {$[\mathrm{Si} / \mathrm{Fe}]$} & {$[\mathrm{Zn} / \mathrm{Fe}]$} & {$[\mathrm{Mn} / \mathrm{Fe}]$} & {$[\mathrm{Ti} / \mathrm{Fe}]$} & {$[\mathrm{Mg} / \mathrm{Fe}]$} \\
\hline Q0100+13 & 2.309 & $-1.78 \pm 0.08$ & $+0.35 \pm 0.07^{\star}$ & $+0.25 \pm 0.04$ & - & - & $+0.44 \pm 0.19$ \\
\hline Q13 & 1.776 & $-2.01 \pm 0$ & $+0.61 \pm 0$ & $+0.75 \pm$ & $0.15 \pm$ & $<-0.45$ & $+0.83 \pm 0.22$ \\
\hline Q2231-00 & 2.066 & $-1.20 \pm 0.09$ & $+0.40 \pm 0.05$ & $+0.41 \pm 0.07$ & $-0.16 \pm 0.10$ & $+0.70 \pm 0.09$ & - \\
\hline $\mathrm{Q} 2343+12$ & 2.431 & $-1.19 \pm 0.06$ & $+0.54 \pm 0.05$ & $+0.62 \pm 0.06$ & $-0.21 \pm 0.05$ & - & - \\
\hline
\end{tabular}

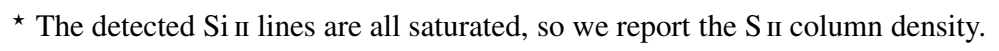

Abundances relative to the solar values of Grevesse et al. (1996).

The $[\mathrm{Fe} / \mathrm{H}]$ ratios correspond to the total metallicities, whereas the $[\mathrm{X} / \mathrm{Fe}]$ ratios are computed by summing only the column densities of the components detected both in the $\mathrm{X}_{\text {II }}$ and Fe II profiles.

the solid phase (Savage \& Sembach 1996). Several pieces of evidence show that some dust is indeed present in DLAs with a dust-to-gas ratio between 2 to $25 \%$ of the Galactic value (Pei et al. 1991; Vladilo 1998). The presence of dust hence implies a depletion of refractory elements (e.g. $\mathrm{Si}, \mathrm{Fe}, \mathrm{Cr}, \mathrm{Ni}$ ) preferentially incorporated into dust grains.

In such a mixed situation (nucleosynthesis plus dust), the analysis of DLA chemical histories is severely limited by the low number of routinely observed elements: $\alpha$-element $\mathrm{Si}$ and $\mathrm{Fe}$-peak element $\mathrm{Fe}$. Occasionally the very important nonrefractory $\alpha$-elements $\mathrm{O}, \mathrm{S}$ and $\operatorname{Ar}$ (located in the Ly $\alpha$ forest) and more frequently the non-refractory $\mathrm{Fe}$-peak element $\mathrm{Zn}$ are detected. We show in this paper that $\mathrm{Mn}, \mathrm{Ti}$ and $\mathrm{Mg}-\mathrm{Fe}$-peak and $\alpha$-elements respectively - are additional elements which can provide important clues to the nature of the DLA star formation history. Well studied in Galactic stars (Nissen et al. 2000; Prochaska \& McWilliam 2000; Chen et al. 2000), relatively little attention has been granted to these elements in DLAs due to their difficult detections (for $\mathrm{Mn}$ see $\mathrm{Lu}$ et al. 1996; Pettini et al. 2000; Ledoux et al. 2002 and for Ti see Prochaska \& Wolfe 1999, 2002; Ledoux et al. 2002). Ratios of $\mathrm{Ti}$ and $\mathrm{Mn}$ relative to $\mathrm{Fe}$ are of special importance because differential depletion and nucleosynthesis tend to work in the opposite sense. Therefore, these ratios help alleviate the dust/nucleosynthesis degeneracy inherent to many other ratios (Prochaska \& Wolfe 2002). Meanwhile, Mg is a refractory $\alpha$ element and a comparison of its abundance with $\mathrm{Si}, \mathrm{S}$ and $\mathrm{O}$ provides greater insight into the dust depletion pattern inherent to DLAs.

We report here three new Mn measurements in DLAs at $z_{\text {abs }} \sim 2$ in addition to the two previous measurements of $\mathrm{Lu}$ et al. (1996) and Ledoux et al. (2002) at similar redshifts, two new $\mathrm{Mg}$ measurements representing the only $\mathrm{Mg}$ measurements in addition to the single previous measurement of Srianand et al. (2000), and the first Ti measurements from Ti II lines at $\lambda_{\text {rest }}>3000 \AA$ in DLAs at $z_{\text {abs }} \sim 2$.

In Sect. 2 we briefly review our observations. In Sect. 3 we describe how we have succeeded to measure the column densities of the weak lines we are interested in and we show the results. In Sect. 4, we highlight the importance of our measurements by discussing individually element by element.

\section{Observations}

We used the unique capability of the Ultraviolet-Visual Echelle Spectrograph UVES (D'Odorico et al. 2000) on the VLT $8.2 \mathrm{~m}$
ESO telescope at Paranal, Chile, to obtain high resolution spectra in the UV $\lambda 3150-4500 \AA$ and in the near-IR $\lambda 6700$ $10000 \AA$ of four relatively bright quasars - Q0100+13, Q1331+17, Q2231-00 and Q2343+12 - with known $z_{\mathrm{abs}}=$ 1.7-2.5 DLAs. Exposure times of 7200-15700 s have been obtained per object. Slit widths of 1 arcsec in the UV and of 0.9 arcsec in the near-IR were fixed throughout the observations with a CCD binning of $2 \times 2$ resulting in a resolution of $F W H M \simeq 6.9 \mathrm{~km} \mathrm{~s}^{-1}$ and $6.4 \mathrm{~km} \mathrm{~s}^{-1}$, respectively. The spectra were reduced using the UVES data reduction pipeline implemented in the ESO MIDAS package (Ballester et al. 2000), and we made a systematic check of each step of the pipeline reduction to make sure of the best result. An average signal-to-noise ratio per pixel of $\sim 30,55$ and 45 was achieved at $\lambda \sim 3700 \AA$, $7500 \AA$ and $9000 \AA$, respectively.

By combining these UVES-VLT spectra with the existing HIRES-Keck spectra obtained by Prochaska \& Wolfe (1999) we cover the total spectral range from 3150 to $10000 \AA$ for the four observed quasars and measure for the first time the column densities of over 15 ions and 12 elements for each of their intervening DLAs. Such a large amount of information allows one to constrain the dust and photoionization effects in the studied DLAs, and thus to infer the likely star formation history of the galaxies associated with these DLAs. The comprehensive analysis of the spectra and the full interpretation of the elemental abundances of the four DLAs will be presented in a future paper (Dessauges-Zavadsky et al., in prep.). Here we focus on the Mn, Ti and Mg measurements in the UVES-VLT spectra and on their importance to understand the intrinsic abundance pattern in DLAs.

\section{Data analysis and results}

The detection of Mn II, Ti II and $\mathrm{Mg}$ II lines is particularly challenging at high redshift because the Mn II lines, the strongest $\mathrm{Ti}$ II lines and the $\mathrm{Mg}$ II doublet are at high rest-wavelengths, $\lambda_{\text {rest }}=2576,2594,2606 \AA, \lambda_{\text {rest }}>3000 \AA$ and $\lambda_{\text {rest }} \approx 2800 \AA$, respectively. The $\mathrm{Mg}$ abundance measurement is hampered by an additional difficulty, the usual saturation of the doublet. The Mg II $\lambda 1239,1240$ lines - generally found in the red wing of the damping Ly $\alpha$ profile - constitute the only possibility to measure the $\mathrm{Mg}$ abundance. Moreover, since all these lines are weak, Mn II and Ti II lines can be easily blended with the numerous telluric absorption lines present at the near-IR wavelengths, whereas $\mathrm{Mg}$ II lines can be contaminated by the Ly $\alpha$ forest. The presence and analysis of other and stronger metal 
lines in the same DLA hence play an important role in determining whether lines are blended and in distinguishing very weak features from the noise.

We used a $\chi^{2}$ minimization routine fitlyman (Fontana \& Ballester 1995) in MIDAS to fit Voigt profiles to the observed DLA absorption lines well described as a complex of components, each defined by a redshift $z$, a Doppler parameter $b$, a column density $N$ and the corresponding errors. Metal species with similar ionization potentials (e.g. neutral and singly ionized species) can be fitted using identical component fitting parameters, i.e. the same $b$ (assuming that the macroturbulent motions dominate over thermal broadening) and the same $z$ in the same component of all metal species and allowing for variations from metal species to metal species in $N$ only. We used relatively strong (but not saturated) lines to fix the component fitting parameters ( $b$ and $z$ ), and excellent profile fits can then be achieved for weak lines and for lines from the Ly $\alpha$ forest where the probability of blending is high by allowing only the column density to vary. This method is the most accurate one to measure the column densities of elements like $\mathrm{Mn}, \mathrm{Ti}$ and $\mathrm{Mg}$.

The results are summarized in Table 1 and the fitting solutions are shown in Figs. 1-4. We had a sufficient number of metal line profiles (on average 6 lines) to well constrain the fitting parameters in the studied DLAs exhibiting multicomponent velocity structures. The telluric lines have been identified thanks to the spectra of a hot, fast rotating star taken in the same nights as the scientific exposures. The $\mathrm{Mg}_{\text {II }} \lambda 1239,1240$ lines detected in the DLA Ly $\alpha$ damping line red wing have been measured after a local renormalization of the spectrum around the $\mathrm{Mg}_{\text {II }}$ lines with the fit of the Ly $\alpha$ damping wing profile. The errors on the measured $\mathrm{Mg}$ column densities have been estimated by varying the continuum level by $5 \%$.

The $[\mathrm{Fe} / \mathrm{H}]$ ratios given in Table 1 correspond to the total metallicity measured in the DLA systems obtained by summing the column densities of all the detected velocity components in the Fe II profiles. The $[\mathrm{X} / \mathrm{Fe}]$ ratios with $\mathrm{X}=\mathrm{Si}, \mathrm{Zn}, \mathrm{Mn}, \mathrm{Ti}$ and $\mathrm{Mg}$, on the other hand, were computed by summing only the column densities of the components detected both in the $\mathrm{X}_{\text {II }}$ and Fe II profiles. In this way we avoid an overestimation of the abundance of $\mathrm{Fe}$ relative to other metal species obtained from weaker line transitions in which generally only the strongest components are detected. In the case of very weak lines, like the Ti II lines, one can indeed underestimate the $[\mathrm{X} / \mathrm{Fe}]$ ratios by up to $0.3-0.4$ dex by considering the total $\mathrm{Fe}$ abundance. In the four studied DLAs this effect is particularly important in the DLAs toward Q2343+12 and Q2231-00 which show complex metal line profiles with a large number of components. The same approach has already been used by Ledoux et al. (2002).

A component by component analysis of the metal ratios in individual DLA systems is interesting because it can reveal spatial fluctuations in the relative abundances (e.g. different chemical composition, different depletion level). We are not going to carry out this type of analysis in this paper, since, due to the weakness of the $\mathrm{Mn}, \mathrm{Ti}$ and $\mathrm{Mg}$ lines, the results would not be reliable. We plan to discuss the possible component to component variations in a future paper where all detected element ratios will be presented (Dessauges-Zavadsky et al., in prep.).

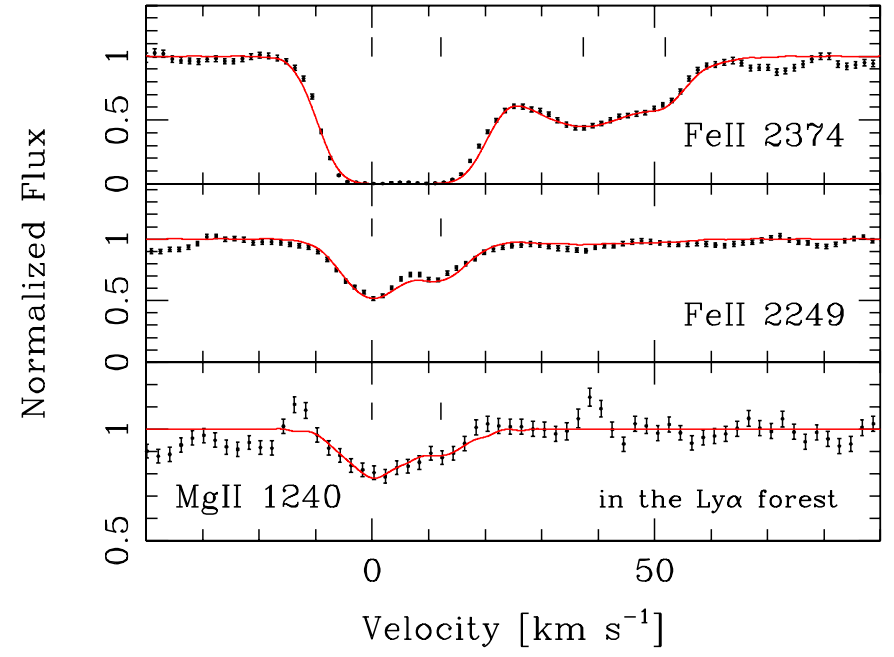

Fig. 1. Velocity plots of the metal line transitions (normalized intensities shown by dots with $1 \sigma$ error bars) for the DLA toward Q0100+13. The zero velocity is fixed at $z=2.309027$. The vertical bars mark the positions of the fitted velocity components and the symbols $\oplus$ correspond to the telluric lines.

\section{1. $Q 0100+13, z_{a b s}=2.309$}

This system called also PHL 957 was carefully studied in Wolfe et al. (1994) and subsequently in Prochaska \& Wolfe (1999) and Prochaska et al. (2001). We have obtained a very similar $\log N(\mathrm{HI})$ column density of $21.37 \pm 0.08$. We confirm their column density measurement of $N\left(\mathrm{Fe}^{+}\right)$obtained in the UVES spectra from the Fe II $\lambda 2249,2260,2344,2374$ lines which were not present in the HIRES spectra. We also agree with their column density measurement of $N\left(\mathrm{Zn}^{+}\right)$. We now present a measurement of the $\mathrm{Mg}$ abundance from the $\mathrm{Mg}_{\text {II }} \lambda 1240$ line located in the red wing of the DLA Ly $\alpha$ profile, the $\mathrm{Mg}$ II $\lambda 1239$ line being blended with Ly $\alpha$ clouds. Molaro et al. (1998) looked for the same $\mathrm{Mg}$ II line, but it was not detected in their low-resolution spectra. Figure 1 shows the Fe II and Mg II profiles where the local continuum around $\mathrm{Mg}^{+}$was defined with the fit of the damping profile of the Ly $\alpha$ line. The Mn II and Ti II lines in this DLA are outside the wavelength coverage of our spectra.

\section{2. $Q 1331+17, z_{a b s}=1.776$}

Many analysis of the damped system toward the very bright quasar Q1331+17 ( $V=16.7)$ have been conducted. We mention here the two most accurate and recent ones of Prochaska \& Wolfe (1999) and Prochaska et al. (2001). In these two papers the authors have assumed the $N(\mathrm{HI})$ measured by Pettini et al. (1994) from relatively low-resolution spectra (1.3 $\AA$ ). We have obtained a new measurement of $\log N\left(\mathrm{H}_{\mathrm{I}}\right)=21.14 \pm 0.08$ from the UVES spectra and confirm that the DLA Ly $\alpha$ line is blended with another strong absorption system at $z_{\mathrm{abs}}=1.786$ with $\log N\left(\mathrm{H}_{\mathrm{I}}\right)=19.80 \pm 0.10$.

We agree with their $\mathrm{Fe}^{+}$column density measurement obtained in the UVES spectra from the Fe II $\lambda 2586$ line not observed in the HIRES spectra. We also confirm the revised $N\left(\mathrm{Zn}^{+}\right)$value of Prochaska et al. (2001) obtained by taking into 


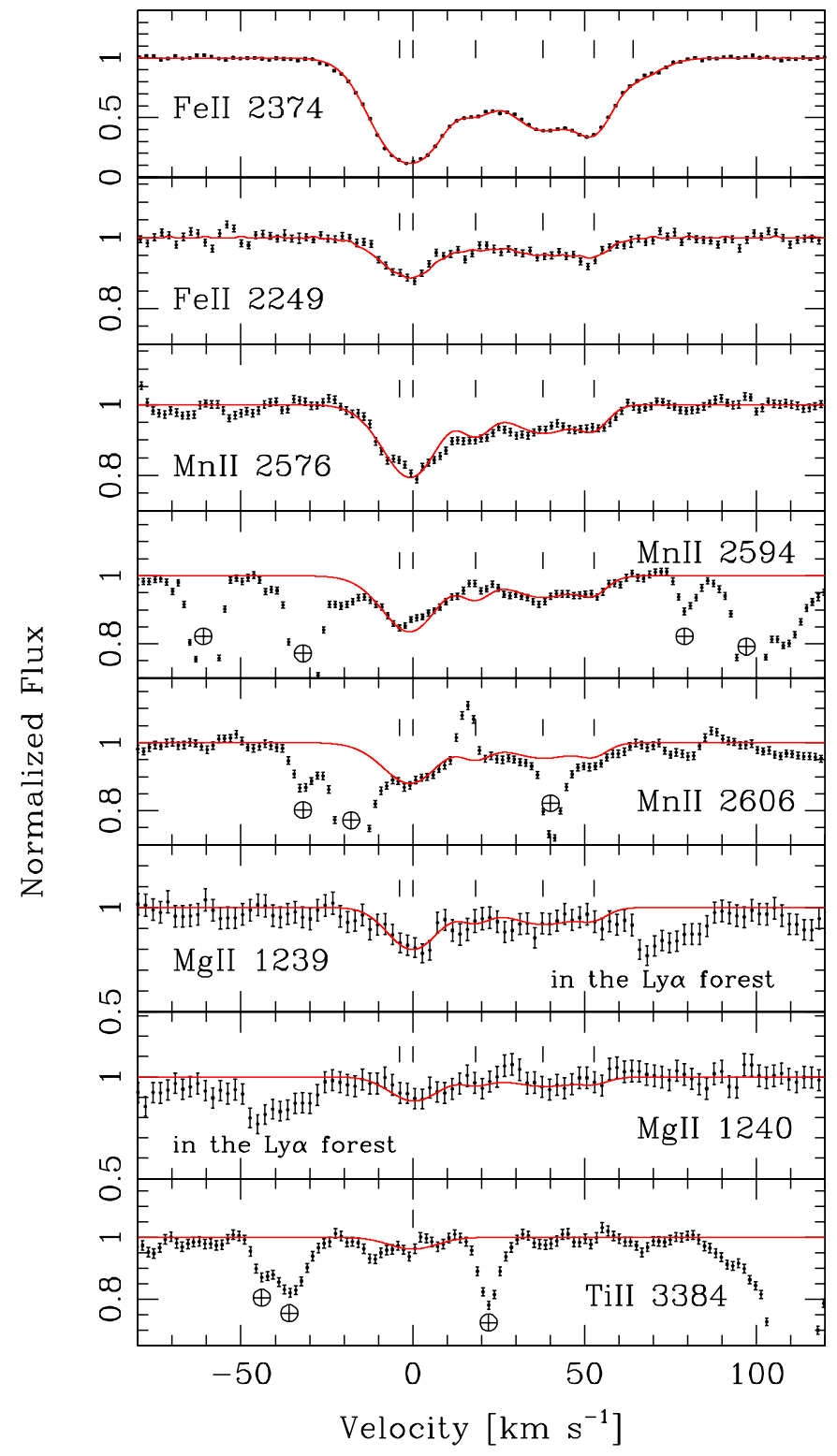

Fig. 2. Same as Fig. 1 for the DLA toward Q1331+17. The zero velocity is fixed at $z=1.776370$.

account the contamination of $\mathrm{Zn}$ II $\lambda 2026$ from the $\mathrm{Mg}_{\mathrm{I}} \lambda 2026$ profile. The UVES spectra allowed us to accurately measure this contamination thanks to the observations of the relatively strong line of $\mathrm{Mg}_{\mathrm{I}}$ 12852. With $[\mathrm{Zn} / \mathrm{Fe}]=0.75 \pm 0.05$, this system exhibits one of the largest $\mathrm{Zn} / \mathrm{Fe}$ ratios (and hence dust depletion level) of any DLA.

We present here new measurements of some transitions not covered in the HIRES spectra, notably Mn II $\lambda 2576,2594,2606$, Mg II $\lambda 1239,1240$ and Ti II $\lambda 3384$ which is the strongest Ti II transition, shown in Fig. 2 with the Fe II profiles. The access to three Mn II lines has allowed us to obtain an accurate measurement of its abundance, although these lines are located in a relatively heavily contaminated area by telluric lines and, in addition, the Mn II $\lambda 2594$ and 2606 lines are blended with some emission from not fully subtracted cosmic rays at $\sim 18$ and $15 \mathrm{~km} \mathrm{~s}^{-1}$, respectively. The normalization in the region of

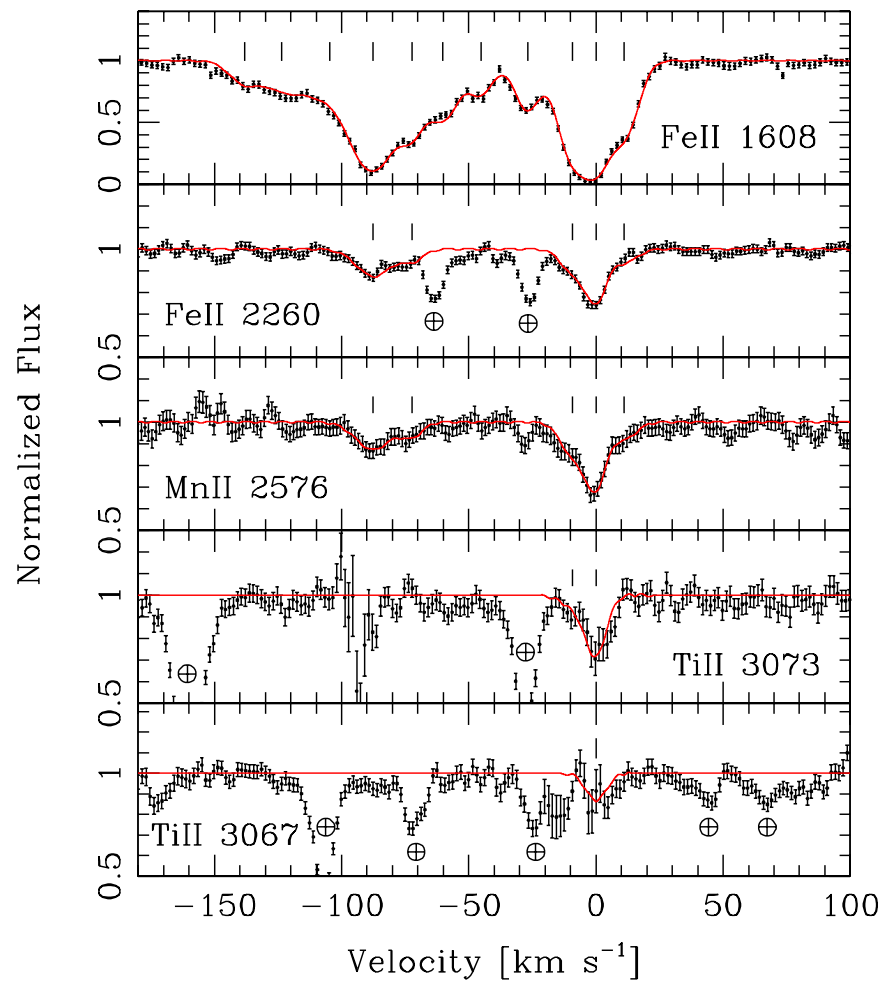

Fig. 3. Same as Fig. 1 for the DLA toward Q2231-00. The zero velocity is fixed at $z=2.066161$.

the $\mathrm{Mg}$ doublet is not optimal over the entire range (specifically around the $\mathrm{Mg}$ II $\lambda 1239$ line), but the adopted error on the $\mathrm{Mg}^{+}$ column density takes this well into account. The detection of the Ti II $\lambda 3384$ line is only marginal, and hence we adopt the measured Ti column density as an upper limit. The value we obtained is more constraining than the higher value deduced by Prochaska et al. (2001) from the Ti II $\lambda 1910$ lines having a 3 times lower oscillator strength.

\section{3. $Q 2231-00, Z_{a b s}=2.066$}

This DLA system has previously been observed and analyzed by Lu et al. (1996) and Prochaska \& Wolfe (1999), and recently completed by Prochaska et al. (2001). Throughout their analysis they have adopted the $\mathrm{H}$ i column density measured by Lu \& Wolfe (1994). We have obtained here a new value for the $\log N\left(\mathrm{HI}_{\mathrm{I}}\right)$ of $20.53 \pm 0.08$. We confirm their Fe II column density measurement and obtain an even more accurate measurement of $N\left(\mathrm{Fe}^{+}\right)$by combining the Fe II $\lambda 1608,1611$ lines detected in the HIRES spectra and the Fe II $\lambda 2260,2344,2374$ lines observed in the UVES spectra. We also confirm their $N\left(\mathrm{Zn}^{+}\right)$measurement.

We now present a measurement of the Mn abundance and a new measurement of the Ti abundance obtained from the newly detected Ti II $\lambda 3067$ and $\lambda 3073$ lines. The Mg II $\lambda 1239,1240$ lines are unfortunately heavily blended with the Ly $\alpha$ lines. Figure 3 shows the Mn II and Ti II profiles with the Fe II profiles. Only the Mn II $\lambda 2576$ transition - the strongest Mn II transition - is covered in our UVES spectra. The anomalously strong feature at about $-30 \mathrm{~km} \mathrm{~s}^{-1}$ in the Mn II profile was not fitted 


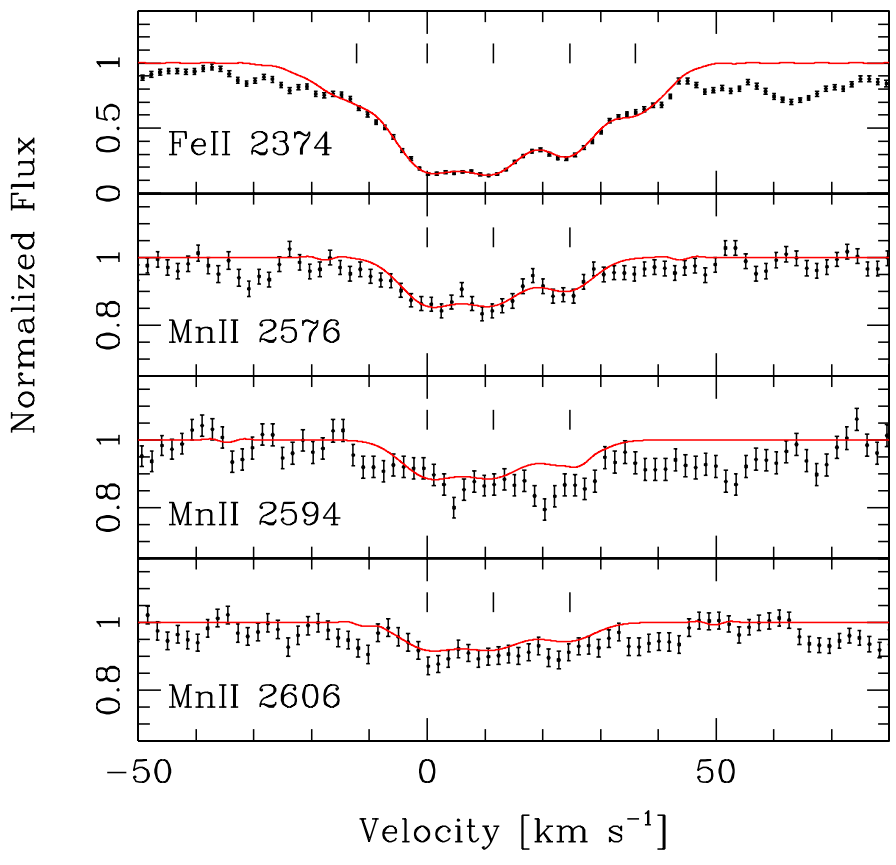

Fig. 4. Same as Fig. 1 for the DLA toward Q2343+12. The zero velocity is fixed at $z=2.431157$. Only a part of the metal line profiles extending over $300 \mathrm{~km} \mathrm{~s}^{-1}$ is shown here, corresponding to the velocity interval where the weak lines are detected, i.e. the velocity interval of the strongest components.

because it is unlikely that it is an absorption from $\mathrm{Mn}$ II, since it appears to be at the noise level in this region of the spectrum (see for instance the features at the same intensities at -140 , $+50,+80 \mathrm{~km} \mathrm{~s}^{-1}$ ). Moreover, other $[\mathrm{X} / \mathrm{Fe}]$ ratios do not show any component at this velocity with a peculiar abundance behavior or dust depletion level relatively to the measured $[\mathrm{X} / \mathrm{Fe}]$ ratios in the other velocity components (Dessauges-Zavadsky et al., in prep.). The measured value for the $\mathrm{Ti}^{+}$column density of $\log N($ Ti iI $)=12.66 \pm 0.08$ is higher than the value obtained by Prochaska \& Wolfe (1999) from the Ti II $\lambda 1910$ lines. Both the detection of $\mathrm{Ti}^{+}$and the measurement of $N\left(\mathrm{Ti}^{+}\right)$presented here are more reliable and accurate, because the signal-to-noise in the UVES spectra is better and, in addition, the oscillator strength of the Ti II $\lambda 3073$ line is slightly higher.

\section{4. $Q 2343+12, z_{a b s}=2.431$}

This system has never been previously studied in detail, only Lu et al. (1998) published the abundance measurements of some elements. We now present the Fe and Mn abundances, the $\mathrm{Ti}$ II and $\mathrm{Mg}$ II lines being outside the wavelength coverage of our UVES spectra, and in a future paper we will provide a complete analysis of all elements observed in this DLA (Dessauges-Zavadsky et al., in prep.). The absorption profiles of this DLA are characterized by a complex structure composed of numerous velocity components (22) and extended over $300 \mathrm{~km} \mathrm{~s}^{-1}$ in velocity space. Figure 4 shows the Fe II and $\mathrm{Mn}$ II profiles plotted over $\sim 80 \mathrm{~km} \mathrm{~s}^{-1}$ - corresponding to the velocity interval of the strongest components and hence to the velocity interval where the weak lines, like the Mn II and

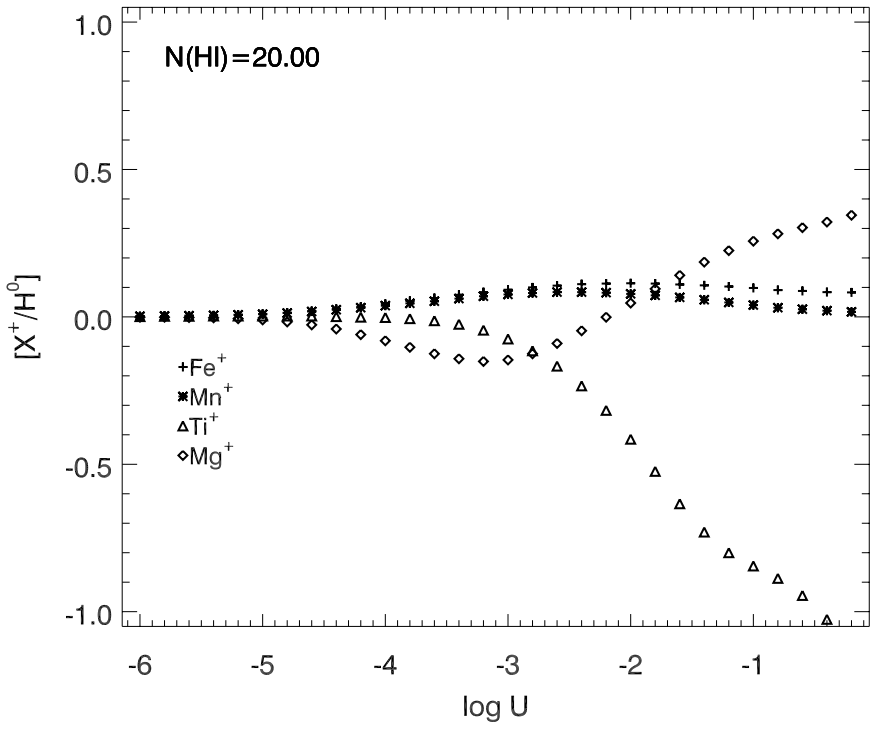

Fig. 5. Predicted logarithmic abundance of several ions $\mathrm{X}^{+}$relative to $\mathrm{H}^{0}$ relative to the intrinsic abundance of these two elements versus a range of ionization parameter $U$, assuming $\log N\left(\mathrm{H}_{\mathrm{I}}\right)=20.0$, a metallicity $[\mathrm{Fe} / \mathrm{H}]=-1$, and the Haardt-Madau EUVB spectrum at $z=2.5$. Departures of $\left[\mathrm{X}^{+} / \mathrm{H}^{0}\right]$ from zero indicate that photoionization corrections are required to calculate intrinsic elemental abundance ratios from the low-ion column densities. Positive (negative) $\left[\mathrm{X}^{+} / \mathrm{H}^{0}\right]$ values would imply overestimates (underestimates) of $[\mathrm{X} / \mathrm{H}]$ from the low-ion ratios. Photoionization corrections are small for all of the elements except $\mathrm{Ti}^{+}$which requires a large positive correction at larger $U$ values.

the $\mathrm{Zn}$ II lines, are detected. The $\mathrm{Fe}^{+}$column density contained in this velocity interval corresponds to only $\sim 75 \%$ of the total $\log N(\mathrm{Fe}$ II $)=14.66 \pm 0.03$. This shows the importance when computing the $[\mathrm{Zn} / \mathrm{Fe}]$ and $[\mathrm{Mn} / \mathrm{Fe}]$ ratios (Table 1 ) to consider only the column densities of velocity components detected both in the $\mathrm{Zn}$ II and Fe II profiles and in the Mn II and Fe II profiles to avoid an underestimation of the abundances of elements obtained from weak lines relative to Fe.

The DLA Ly $\alpha$ line is outside our wavelength coverage, therefore we assume the $\log N\left(\mathrm{H}_{\mathrm{I}}\right)=20.35 \pm 0.05$ measured by D'Odorico et al. (2002). This H I column density is relatively low and it is hence important and interesting to discuss the influence of ionization on the abundance pattern in this DLA. Here we present the effects of photionization on $\mathrm{Mn}, \mathrm{Ti}$ and $\mathrm{Mg}$ relative to $\mathrm{Fe}$ for physical conditions relevant to the damped Ly $\alpha$ systems (see also Vladilo et al. 2001). Figure 5 shows that for $\mathrm{Mn}$ and $\mathrm{Mg}$ the effects are small for nearly any assumptions on the ionizing radiation, but we find that one could underestimate $\mathrm{Ti} / \mathrm{Fe}$ in some DLAs having a high ionizing parameter.

\section{Discussion}

In the following discussion we interpret our results on the Mn, $\mathrm{Ti}$ and $\mathrm{Mg}$ abundances and underline their complementarity to the measurements of other elements.

Manganese. Major recent studies of Mn abundances in the Milky Way have been completed by Nissen et al. (2000) and Prochaska \& McWilliam (2000). About 120 [Mn/Fe] 
measurements from $\mathrm{F}$ and $\mathrm{G}$ stars in the metallicity interval $-1.4<[\mathrm{Fe} / \mathrm{H}]<0.1$ have been obtained. Their results confirmed that $\mathrm{Mn}$ behaves in an opposite sense to the $\alpha$-elements: a steady decline to $[\mathrm{Mn} / \mathrm{Fe}] \sim-0.15$ from solar metallicity to $[\mathrm{Fe} / \mathrm{H}] \sim-0.7$ and a possible drop in $[\mathrm{Mn} / \mathrm{Fe}]$ below $[\mathrm{Fe} / \mathrm{H}] \sim-0.7$. Mn is an Fe-peak element produced by massive stars and mostly by SNIa. Its behavior is not completely understood, although it is relatively well accounted for by metallicity-dependent yields for massive stars and for SNIa, illustrating a nice example of the "odd-even" effect for the Fenuclei (Goswami \& Prantzos 2000).

Although $\mathrm{Mn}$ is a refractory element, the $\mathrm{Mn} / \mathrm{Fe}$ ratio is an important and useful diagnostic ratio. Indeed, from Savage \& Sembach (1996) we can see that Mn and Fe have nearly identical depletion levels in the warm halo ISM clouds (the physical environment whose depletion level is most likely similar to the DLAs) and Mn is less depleted than Fe in the cold ISM clouds. Therefore, as stressed by Lu et al. (1996), sub-solar [Mn/Fe] ratios in gas-phase abundances must be interpreted in terms of nucleosynthesis while super-solar $[\mathrm{Mn} / \mathrm{Fe}]$ ratios would require significant dust depletion.

The three new $[\mathrm{Mn} / \mathrm{Fe}]$ measurements in the DLAs at $z_{\mathrm{abs}} \sim$ 2 (see Table 1) are in agreement with the $[\mathrm{Mn} / \mathrm{Fe}]$ measurements in DLAs at $z_{\text {abs }}<2$ (Lu et al. 1996; Lopez et al. 1999; Pettini et al. 1999, 2000; Ledoux et al. 2002), and confirm that $\mathrm{Mn}$ is underabundant in galaxies associated with DLAs in a very similar way to Galactic stars (see Fig. 6). We also note that the DLA system toward Q1331+17 exhibits a much larger $\mathrm{Mn} / \mathrm{Fe}$ ratio $-[\mathrm{Mn} / \mathrm{Fe}]=-0.15 \pm 0.04$ at a metallicity of $[\mathrm{Zn} / \mathrm{H}]=-1.27 \pm 0.09([\mathrm{Fe} / \mathrm{H}]=-2.01 \pm 0.09)-$ than the observed values in the Galactic stars at the same metallicity consistent with the fact that this DLA has a large depletion level.

The nice agreement between the $\mathrm{Mn} / \mathrm{Fe}$ ratios in DLAs and in Galactic stars seen in Fig. 6 with a very comparable trend with decreasing metallicity, but a greater scatter in DLAs, suggests that the DLAs and the Milky Way share some similarities in their star formation histories. However, although the greater scatter may be explained by measurement uncertainties and the effects of differential depletion, these similarities must be cautiously interpreted and investigated in light of all of the elements observed in the DLAs. For example, it remains unclear what fraction of the damped systems exhibit $\alpha$ enhancements relative to Fe-peak elements consistent with the observations in Galactic metal-poor stars (e.g. Vladilo 1998; Centurión et al. 2000; Prochaska \& Wolfe 2002). These similarities/discrepancies between the abundance patterns in DLAs and in Galactic stars have to be further carefully analyzed. They emphasize the importance of studying the $\mathrm{Mn} / \mathrm{Fe}$ ratios in DLAs in addition to the $\alpha / \mathrm{Fe}$ ratios to better define the differences in the star formation histories of the DLAs and our Galaxy. Moreover, since direct observations of DLAs show a wide range of galactic morphological types (e.g. Le Brun et al. 1987), it is important to examine variations within their abundance patterns to search for variations in their star formation histories. Finally, by obtaining Mn measurements in low metallicity DLAs one can test nucleosynthetic models of
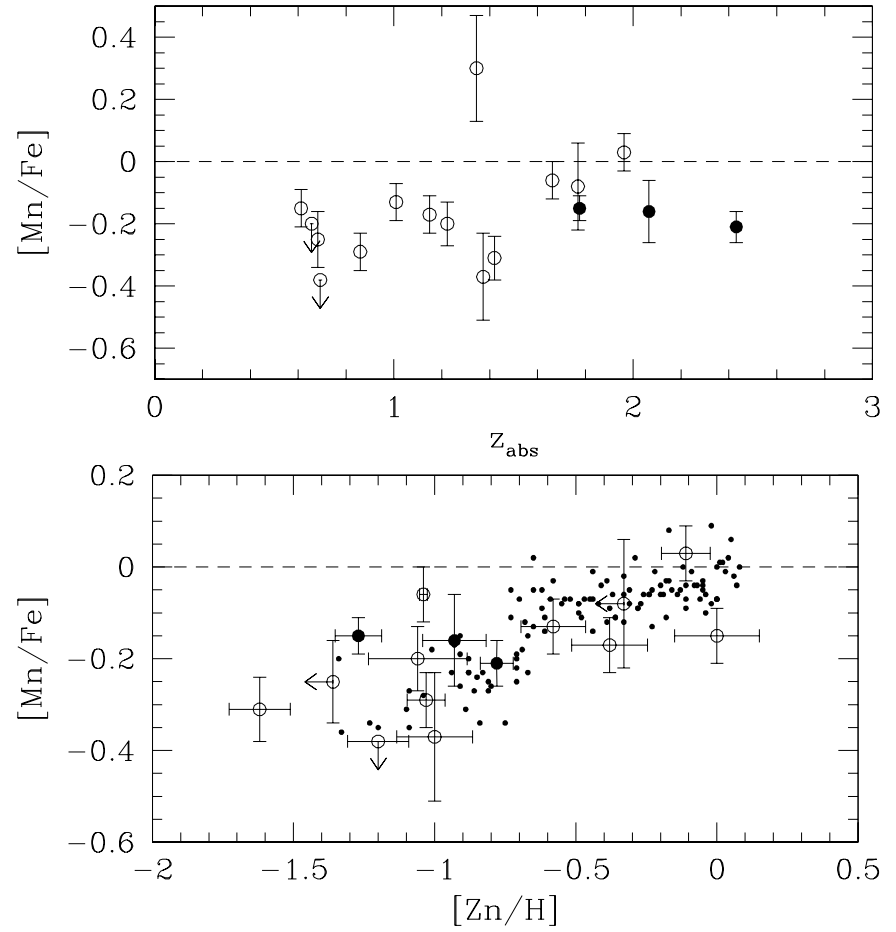

Fig. 6. Relative abundances of $[\mathrm{Mn} / \mathrm{Fe}]$ versus $z_{\text {abs }}$ of the DLA systems (upper panel) and versus the abundances of $\mathrm{Zn}$, i.e. metallicities (lower panel). The open circles are literature values (Lu et al. 1996; Lopez et al. 1999; Pettini et al. 1999, 2000; Ledoux et al. 2002) which have been corrected from a possible underestimation due to a larger number of detected $\mathrm{Fe}$ II components relative to the $\mathrm{Mn}$ II components (see Sect. 3) when a detailed profile modeling was available. The filled circles are our measurements presented here. We have included to this sample only the DLAs for which a direct $\mathrm{H}_{\text {I }}$ column density measurement is known and we assume that in DLAs the metallicity $[\mathrm{Zn} / \mathrm{H}] \approx$ $[\mathrm{Fe} / \mathrm{H}]$ in absence of dust. The dots are $[\mathrm{Mn} / \mathrm{Fe}]$ versus $[\mathrm{Fe} / \mathrm{H}]$ values measured in Galactic stars from Prochaska \& McWilliam (2000).

Mn production - major source of Mn: SNIa or SNII with strong metallicity dependence of the yield.

Titanium. Many Ti stellar abundance measurements exist in our Galaxy over a wide metallicity interval $-4.0<[\mathrm{Fe} / \mathrm{H}]<$ 0.5 (e.g. Chen et al. 2000). Ti is generally accepted as an $\alpha$ element, because it exhibits abundance trends similar to other $\alpha$-elements, although this behavior has not been reproduced by chemical evolution models (Timmes et al. 1995; Goswami \& Prantzos 2000).

Thus far, only a few Ti measurements exist in DLAs at $z_{\text {abs }}<1.5$ (Ledoux et al. 2002), and efforts to measure Ti in DLAs at $z_{\mathrm{abs}}>1.5$ have focused on the pair of very weak lines at $\lambda_{\text {rest }} \approx 1910 \AA$ and have yielded only a few tenuous detections (e.g. Prochaska \& Wolfe 1997, 1999; Prochaska et al. 2001). Our observations of the stronger Ti II $\lambda 3073,3242,3384$ lines resulted in one robust detection and one significant upper limit.

$\mathrm{Ti}$ is a refractory element and exhibits an equivalent or higher depletion level in the ISM clouds than Fe (Howk et al. 1999). This runs contrary to the expectation for an $\alpha$ enhancement and, therefore, positive departures of [Ti/Fe] from the solar ratio is evidence for an $\alpha$-enhancement in the system 
independently of the presence of dust. Negative [Ti/Fe] ratios on the contrary should provide evidence for dust depletion. We found in the DLA toward Q2231-00, $[\mathrm{Ti} / \mathrm{Fe}]=+0.70 \pm 0.09$ which suggests a dominance of type II SNe relatively well consistent with the ratio of $[\mathrm{S} / \mathrm{Zn}]=0.17 \pm 0.09$ (DessaugesZavadsky et al., in prep.). In the DLA toward Q1331+17, on the other hand, $[\mathrm{Ti} / \mathrm{Fe}]<-0.45$ suggests significant dust depletion in agreement with $[\mathrm{Zn} / \mathrm{Fe}]=+0.75 \pm 0.05$.

Magnesium. $\mathrm{Mg}$ is an $\alpha$-element with a refractory nature whose depletion level is slightly higher than Si but lower than $\mathrm{Fe}$ (Savage \& Sembach 1996). Mg/Fe ratios are useful complements to other $\alpha / \mathrm{Fe}$ ratios measured in the same DLA to deduce information on the star formation history of the galaxy associated with the DLA. Moreover, the study of $\alpha$-element ratios with different depletion levels, like $\mathrm{Si} / \mathrm{Mg}, \mathrm{Mg} / \mathrm{Ti}$ and $\mathrm{Si} / \mathrm{Ti}$, is another very interesting way to highlight the presence of dust in DLAs. But perhaps the most valuable aspect of the Mg measurements relates to the use of Mg equivalent widths to identify low redshift absorption galaxies when the Ly $\alpha$ lines are not detected (e.g. Bergeron \& Boissé 1991; Rao \& Turnshek 2000). It is therefore important to know the range of $\mathrm{Mg}$ column densities in DLAs to confirm the empirical method to recognize them at low redshift.

While interesting for the reasons quoted above, the number of $\mathrm{Mg}$ measurements in DLAs remains tiny. With our two measurements a total of only three $\mathrm{Mg}$ abundance measurements are known in DLAs. They all show positive $[\mathrm{Mg} / \mathrm{S}, \mathrm{Si}]$ ratios of 0.10-0.20 dex (see Table 1 and the DLA system in Srianand et al. 2000), in contradiction to the negative values expected from the differential depletion. But, since the detection of positive $\mathrm{Mg} / \mathrm{S}$, Si ratios is at the level of the measurement errors of $\sim 0.2$ dex and differs from the maximum over-depletion of $\mathrm{Mg}$ relatively to $\mathrm{Si}$ (of $0.2 \mathrm{dex}$ ) observed in the ISM warm gas by only $2 \sigma$ (Welty et al. 1999), it has to be further confirmed by additional $\mathrm{Mg}$ measurements before interpreting it as a peculiar nucleosynthetic effect similar to the observations of some Galactic stars (e.g. McWilliam et al. 1995).

Acknowledgements. The authors wish to extend special thanks to all people working at ESO/Paranal for the high quality of the UVES spectra obtained in service mode. M.D.-Z. is supported by an ESO Studentship and the Swiss National Funds, and J.X.P. acknowledges support through the Hubble Fellowship grant HF-01142.01-A awarded by STScI (NASA).

\section{References}

Ballester, P., Modigliani, A., Boitquin, O., et al. 2000, The Messenger, 101,31

Bergeron, J., \& Boissé, P. 1991, A\&A, 243, 344

Centurión, M., Bonifacio, P., Molaro, P., \& Vladilo, G. 2000, ApJ, 536,540
Chen, Y. Q., Nissen, P. E., Zhao, G., Zhang, H. W., \& Benoni, T. 2000, A\&AS, 141, 491

Dessauges-Zavadsky, M., D’Odorico, S., \& Prochaska, J. X. 2002, in preparation

D’Odorico, S., Cristiani, S., Dekker, H., et al. 2000, in Proc. of SPIE, vol. 4005, Discoveries and Research Prospects from 8- to 10Meter-Class Telescopes, ed. J. Bergeron (Bellingham: SPIE), 121

D’Odorico, V., Petitjean, P., \& Cristiani, S. 2002, A\&A, in press [astro-ph/0205299]

Fontana, A., \& Ballerster, P. 1995, The Messenger, 80, 37

Goswami, A., \& Prantzos, N. 2000, A\&A, 359, 191

Grevesse, N., Noels, A., \& Sauval, A. J. 1996, Astron. Soc. Pac. Conf. Ser., 99, 117

Howk, J. C., Savage, B. D., \& Fabian, D. 1999, ApJ, 525, 253

Le Brun, V., Bergeron, J., Boissé, P., \& Deharveng, J.-M. 1987, A\&A, 321, 733

Ledoux, C., Bergeron, J., \& Petitjean, P. 2002, A\&A, 385, 802

Lopez, S., Reimers, D., Rauch, M., Sargent, W. L. W., \& Smette, A. 1999, ApJ, 513, 598

Lu, L., \& Wolfe, A. M. 1994, AJ, 108, 44

Lu, L., Sargent, W. L. W., Barlow, T. A., Churchill, C. W., \& Vogt, S. S. 1996, ApJS, 107, 475

Lu, L., Sargent, W. L. W., \& Barlow, T. A. 1998, AJ, 115, 55

Matteucci, F. 2001, The chemical evolution of the Galaxy, Astrophysics and Space Science library, 253 (Kluwer Academic Publishers)

McWilliam, A., Preston, G. W., Sneden, C., \& Searle, L. 1995, AJ, 109,2757

Molaro, P., Vladilo, G., \& Centurión, M. 1998, MNRAS, 293, L37

Nissen, P. E., Chen, Y. Q., Schuster, W. J., \& Zhao, G. 2000, A\&A, 353, 722

Pei, Y. C., Fall, S. M., \& Bechtold, J. 1991, ApJ, 378, 6

Pettini, M., Smith, L. J., Hunstead, R. W., \& King, D. L. 1994, ApJ, 426, 79

Pettini, M., Ellison, S. L., Steidel, C. C., \& Bowen, D. V. 1999, ApJ, 510,57

Pettini, M., Ellison, S. L., Steidel, C. C., Shapley, A. E., \& Bowen, D. V. 2000, ApJ, 532, 170

Prochaska, J. X., \& Wolfe, A. M. 1997, ApJ, 474, 140

Prochaska, J. X., \& Wolfe, A. M. 1999, ApJS, 121, 369

Prochaska, J. X., \& McWilliam, A. 2000, ApJ, 537, L57

Prochaska, J. X., Wolfe, A. M., Tytler, D., et al. 2001, ApJS, 137, 21

Prochaska, J. X., \& Wolfe, A. M. 2002, ApJ, 566, 68

Rao, S. M., \& Turnshek, D. A. 2000, ApJS, 130, 1

Savage, B. D., \& Sembach, K. R. 1996, ARA\&A, 34, 279

Srianand, R., Petitjean, P., \& Ledoux, C. 2000, Nature, 408, 931

Timmes, F. X., Woosley, S. E., \& Weaver, T. A. 1995, ApJS, 98, 617

Tinsley, B. M. 1979, ApJ, 229, 1046

Vladilo, G. 1998, ApJ, 493, 583

Vladilo, G., Centurión, M., Bonifacio, P., \& Howk, J. C. 2001, ApJ, 557,1007

Welty, D. E., Hobbs, L. M., Lauroesch, J. T., et al. 1999, ApJS, 124, 465

Wolfe, A. M., Fan, X.-M., Tytler, D., et al. 1994, ApJ, 435, L101

Wolfe, A. M., Lanzetta, K. M., Foltz, C. B., \& Chaffee, F. H. 1995, ApJ, 454, 698 